\title{
The innate immune properties of airway mucosal surfaces are regulated by dynamic interactions between mucins and interacting proteins: the mucin interactome
}

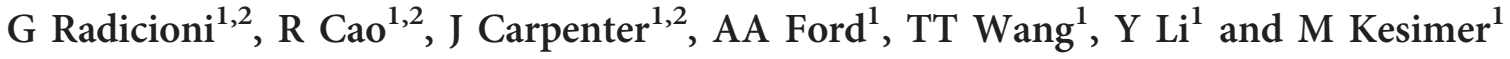

Chronic lung diseases such as cystic fibrosis, chronic bronchitis, and asthma are characterized by hypersecretion and poor clearance of mucus, which are associated with poor prognosis and mortality. Little is known about the relationship between the biophysical properties of mucus and its molecular composition. The mucins MUC5B and MUC5AC are traditionally believed to generate the characteristic biophysical properties of airway mucus. However, the contribution of hundreds of globular proteins to the biophysical properties of mucus is not clear. Approximately one-third of the total mucus proteome comprises distinct, multi-protein complexes centered around airway mucins. These complexes constitute a discrete entity we call the "mucin interactome". The data suggest that while the majority of these proteins interact with mucins via electrostatic and weak interactions, some interact through very strong hydrophobic and/or covalent interactions. Using reagents that interfere with protein-protein interactions, the complexes can be disassembled, and mucus rheology can be dramatically altered. Using MUC5B-glutathione S-transferase (GST) and MUC5B-galectin-3 as a representative of these interactions, we provide evidence that individual mucin protein interactions can alter the biophysical properties of mucus and modulate the biological function of the protein. We propose that the key mechano- and bio-active functions of mucus depend on the dynamic interactions between mucins and globular proteins. These observations challenge the paradigm that mucins are the only molecules that confer biophysical properties of mucus. These observations may ultimately lead to a greater understanding of the system and guide the development of strategies for more effective interventions using better therapeutic agents.

\section{INTRODUCTION}

The airway epithelial mucosal barrier is a major component of the lungs' innate immunity, and is the first line of defense against inhaled physical, chemical, and pathogenic insults. ${ }^{1} \mathrm{~A}$ common protective feature of the epithelium is its innate mechanical defense, which is the ability of mucociliary action and/or cough clearance to scavenge/trap and remove insults. ${ }^{2,3}$ Airway mucus, an essential component of this mechanism, is an integrated, active visco-elastic gel matrix that consists of a complex network of mucins, enzymes, and a wide variety of defense proteins that detect, immobilize, destroy, and/or remove a range of foreign bodies, toxins, and pathological materials. Mucus is produced at low levels in healthy airways. However, in diseases such as chronic bronchitis, ${ }^{4}$ cystic fibrosis, ${ }^{5}$ and asthma, ${ }^{6}$ an abnormal mucus composition and quantity can produce a muco-obstructive disease phenotype.

Mucus, a strong polymer network, is composed of an array of protein biomolecules ranging in molecular weight from approximately $6 \mathrm{kD}$ to $100 \mathrm{MD} .^{7-9}$ These molecules may be split into two distinct groups: the first and major group of molecules consists of globular type proteins with a molecular weight between 6 and $200 \mathrm{kD}$ with a diverse array of proposed

\footnotetext{
${ }^{1}$ Department of Pathology and Laboratory Medicine, Cystic Fibrosis/Pulmonary Research and Treatment Center, Marsico Lung Institute, University of North Carolina at Chapel Hill, NC, USA. Correspondence: M Kesimer (kesimer@med.unc.edu)

${ }^{2}$ These authors contributed equally to this work. 
functions ( $70-80 \%$ by weight); and the second group of molecules consists of mucins ( $20-30 \%$ by weight) that are large space-filling glycoconjugates with a typical molecular weight of $200 \mathrm{kD}$ to $100 \mathrm{MD}$. We have limited information about the visco-elastic properties of airway mucus and how it may be optimized in some cases for removal by cough and in other cases by flow over cilia. The gel-forming mucins of the airway, MUC5B and MUC5AC, are the main gel-forming mucins in cultured airway epithelial cell (human tracheobronchial epithelial (HBE) cell) secretions. ${ }^{1,8}$ The membrane-associated mucins MUC1, 4, 13, 16, and 20 are also present in airway secretions ${ }^{1,10}$ and most likely have as-yet undocumented roles in the properties of normal gels.

Mucins are high-molecular-weight glycoprotein components of mucosal barriers. Mucin genes encode glycoproteins, and O-linked carbohydrates comprise most of their mass. These proteins have a distinctive domain that contains a high percentage of serines, threonines and, generally, prolines. In addition to their large, central, heavily glycosylated region, gel-forming mucins have highly functional and complicated $N$ - and $C$-terminal naked protein regions that mediate oligomerization and the interaction with globular proteins. The molecular architecture of gel-forming mucins, particularly in the $C$ - and $N$-terminal region, is similar to that of the von Willebrand factor. ${ }^{11}$

Because the polymeric gel-forming mucins, MUC5AC and MUC5B, are the major constituents of the airway mucus gel, they are traditionally believed to dominate the biophysical properties of mucus that permit a smooth flow over the ciliated healthy epithelium in a well-hydrated environment. ${ }^{12}$ Contributions of hundreds of globular proteins, however, to these properties have not yet been fully elucidated. Despite substantial research on the subject, it is still not clear whether mucin polymers are the key contributors to the biophysical properties of mucus gels. In an effort to address the normal composition of airway mucus, we observed that at least $30 \%$ of the proteins in HBE secretions and induced sputum are formed by distinct protein complexes centered around mucins. We propose that these complexes constitute a discrete secretory entity we call the "mucin interactome". The basis of these structures and their function(s), e.g., their contribution to the biophysical and biological functions of airway mucus, remains a mystery and is the focus of this study. We hypothesized that mucin-protein interactions dynamically control the critical innate immune functions of the airway mucosal barrier, including its physical (rheological) and biological (antimicrobial, antioxidant) functions. Therefore, as a first step toward testing this hypothesis, primary HBE cells and a broad range of biochemical, biophysical, and proteomic approaches were employed. The aim of this study was to rigorously characterize the airway mucin interactome and study its effects on the basic rheological functions of airway mucus that provide optimal innate airway defense, including hydration, viscosity, and elasticity.

\section{RESULTS}

\section{Characterization of the mucin interactome}

Initial isolation and identification of the mucin interactome utilizing size-exclusion chromatography followed by density-gradient centrifugation. A two-step "associative" isolation procedure was performed to isolate the mucins and their complexes under physiological conditions without chaotropic agents or detergents that would disturb the protein-protein interactions. We first performed a gel filtration chromatography separation of diluted $\mathrm{HBE}$ cell culture secretions in $200 \mathrm{~mm} \mathrm{NaCl}$, employing a Sephacryl 1000 column (Figure 1a). Virtually, all of the carbohydrate identified by the periodic acid/Schiff reaction (pink line) was recovered in the Void $\left(V_{\mathrm{o}}\right)$ of the column. Note that membrane mucins such as MUC1, MUC4, and MUC20 are not large enough to elute in this fraction. Importantly, a substantial amount of the small non-mucin proteins that were assayed by the amido black dye was observed in the $\mathrm{V}_{\mathrm{o}}$ region along with the mucins. Additionally, as expected, most of the amido blank staining, as an indicator of proteins, was observed at $V_{\mathrm{t}}$ (included volume, black line).

The void fractions (fractions 14-18) were pooled and subjected to proteomics analysis. In addition, to distinguish the mucinprotein complexes in the Vo fractions from other protein
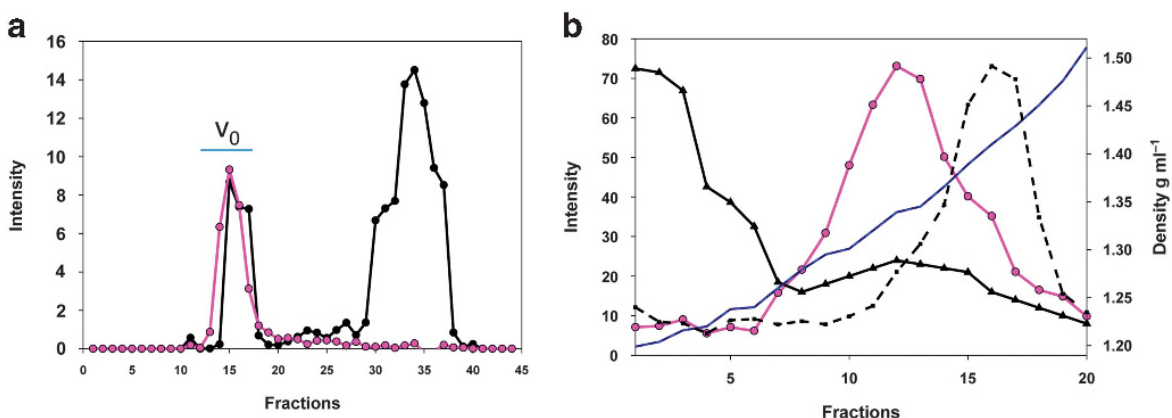

Figure 1 Two-step isolation of the mucin protein complexes from human tracheobronchial epithelial (HBE) secretions. An aliquot (10 ml) of HBE secretions was subjected to gel filtration chromatography on an $\mathrm{S} 1000$ column. Then, 2 -ml fractions were collected and analyzed with periodic acid Schiff (PAS) (pink) and amido black staining (black) (a). The PAS-rich void volume was pooled and subjected to cesium chloride (CsCl) density-gradient centrifugation (b). The fractions were unloaded and probed using slot blotting with PAS (pink) and amido black staining (black) and a keratin sulfate antibody (dotted black). Fractions 1-6, 8-15, and 16-20 were pooled and subjected to proteomic analysis, as reported in Figure 2. The blue line represents density. The intensity units are arbitrary. 
complexes and/or structural organizations, the periodic acid Schiff (PAS)-rich S1000 void fractions were pooled and subjected to isopycnic cesium chloride $(\mathrm{CsCl})$ density-gradient centrifugation at a starting density of $1.45 \mathrm{~g} \mathrm{ml}^{-1}$ as the second step in the isolation. After centrifugation, 2-ml fractions were removed from the tubes beginning at the top, and the fractions were subjected to PAS and amido black analysis to detect mucin and proteins, respectively (Figure 1b). The amido black-rich fractions (1-6), PAS-rich middle fractions (8-15), and the keratan sulfate-rich fractions (16-20) were pooled and subjected to proteomic analysis, as described below.

Identification of the mucin interactome. We previously reported that isolated airway mucins were accompanied by significant number of proteins when isolated in the presence of $4 \mathrm{M} \mathrm{GuHCl}$; i.e., "dissociative conditions". 8 Here, we deliberately used "associative" physiological conditions to avoid chemical disturuption of the protein-protein interactions. Using this approach, we found that at least $1 / 3$ of the total airway mucus proteins co-isolated with mucins. The first step of the $S 1000$ size-exclusion separation and proteomic analysis indicated that in addition to mucins, a wide range of innate defense proteins was detected in the $\mathrm{V}_{0}$. These included LPLUNC1 (BPIFB1), complement C3; the polymeric Ig receptor; DMBT1; lysozymes; and membrane, cytoskeletal, and intracellular proteins, including ezrin, CD133, beta actin, and solute carrier family proteins. The presence of these distinct sets of proteins in the $\mathrm{V}_{0}$, i.e., at sizes substantially larger than their apparent molecular mass, was highly suggestive of the presence of distinct complexes in the S1000 void.

Further isolation and identification of the discrete interactomes was achieved using $\mathrm{CsCl}$ density-gradient centrifugation (Figure 1b). The proteins comprising the pools from the density gradient were identified using mass spectrometry and are presented as an interactome map in Figure 2; and the whole list of the proteins, with details, is provided in the Supplementary Table online. As expected, the low-density pool of the gradient $(T)$ contained most of the globular proteins, including the structural, membrane-related, and calciumbinding proteins and MUC1, which was unexpected. MUC1 was present throughout the gradient, and agarose gel electrophoresis demonstrated mature MUC1 glycoprotein in all cases (data not shown). Because mucins, including small membrane mucins, are highly glycosylated and because their buoyant density is typically higher than $1.3 \mathrm{~g} \mathrm{ml}^{-1}$, the presence of MUC1 in the low-density fractions (top pool) was unexpected. Actin was also located in the "T" region along with membranes and proteins with structural organizing roles, such as ezrin and EBP50. Membrane and channel proteins, such as nuclear chloride ion channel protein 1 (CLIC1) and solute carrier family proteins 9 and 34, were also detected, suggesting the presence of an organized membranous structure in this pool. Further analysis with electron microscopy revealed that these proteins were associated with exosome-like vesicles, as previously reported. ${ }^{13}$
Proteomic analysis of the mucin-rich pool (fractions 8-15) indicated that the pool was dominated by the gel-forming mucins MUC5B and MUC5AC, and revealed approximately 30 globular proteins, including LPLUNC1, PLUNC, WAPdisulfide core protein 4, GST, antileukoprotease, DMBT1, galectins (galectin-3, -8 , and -9), complement component C3, and polymeric IgG receptor (Figure 2). None of these globular proteins have buoyant densities that are consistent with migration into this pool after centrifugation, which had an average density of $1.35 \mathrm{~g} \mathrm{ml}^{-1}$.

The membrane-bound mucins MUC1, MUC4, MUC16, and MUC20 were abundantly present in the high-density region (the bottom pool, fractions 16-20), which had an average density of $1.45 \mathrm{~g} \mathrm{ml}^{-1}$. The gel-forming mucins MUC5B and MUC5AC were also present in this region but at lower levels than those in the middle pool. Globular proteins, such as WAP four disulfide core protein 2 (human epididymis protein 4) and galectins, were also present in the bottom fractions. Antiproteases, such as leucocyte elastase inhibitor (SERPIN B1), anti-leukoprotease, SERPIN B3, and squamous cell carcinoma antigen 1 , were also present in the mucin fractions.

Macromolecular organization of the mucin framework, supported by interacting proteins, using atomic force microscopy. To visually appreciate how interacting partners of the gel-forming mucins contribute to the organizational framework of the mucus gel, isolated mucin-protein complexes were imaged at high resolution using atomic force microscopy (AFM) (Figure 3). A raw topographical 2D high-resolution version of Figure 3 is provided in the Supplementary Material (Supplementary Figure S1). As shown in Figure 3, the primary infrastructure of the network consists mainly of mucin's glycosylated chains with different thicknesses (green strands). Globular proteins/glycoproteins of different sizes (brown nodes, $2-12 \mathrm{~nm}$ in height) together with membrane mucins determine and/or augment the macromolecular organization of the mucin network. For instance, an innate defense protein, likely DMBT1 (by structural prediction ${ }^{14}$ ), is incorporated into the mucin network (Figure 3a). Glycosylated chains of mucins (green strands) are generally found in linear form in the sample. However, the presence a compact/folded form of mucin ${ }^{15}$ characterized by a big globular protein node in its center was also observed (Figure 3b). As can be seen from the figure, in addition to the predominantly individual linear strands, mucin glycosylated domains can also be seen as clustered chains likely via self and/or indirect associations. In contrast, a dissociatively purified MUC5B network displays a different structural organization; with fewer protein nodes, a more linear and uniform distribution of mucin chains with no clustering, and with no compact form present (Supplementary Figure S2).

\section{Disassembling mucin-protein complexes with chaotropic solvents}

The effects of chaotropic solvents on the mucin interactome stability and the mucin macromolecular structure were investigated using SEC-MALLS (multi-angle laser light scattering combined with size-exclusion chromatography), mass 


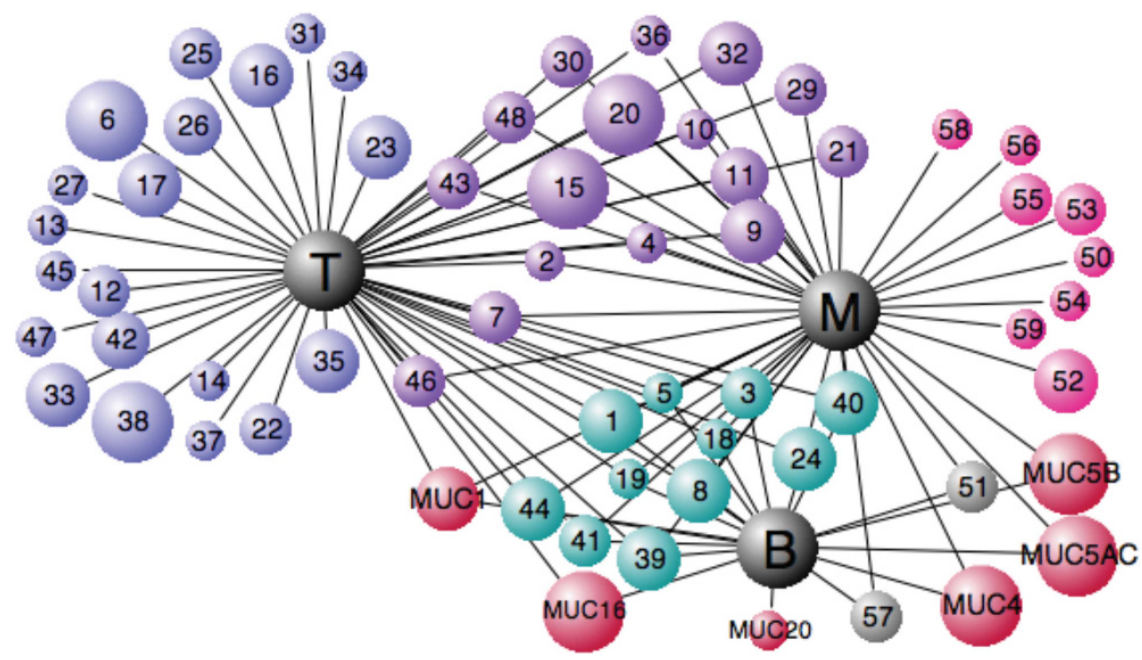

Figure 2 Airway mucin interactome map: human tracheobronchial epithelial (HBE) secretions were first subjected to S1000 size-exclusion chromatography. Mucins and their interacting proteins were then separated from other structures and protein-protein complexes using isopycnic densitygradient centrifugation. The low-density ( $T$, fractions $1-6)$, medium-density ( $M$, fractions $8-15)$, and high-density ( $B$, fractions $16-20)$ regions were pooled and subjected to a proteomics analysis. Magenta: mucins, Red: proteins found only in the "M" region, Purple: proteins found in both the "M" and "T" regions, Blue: proteins only found only in the "T" region, Green: proteins found in all regions. The size of each proteins cycle reflects its relative abundance. The detected proteins are numbered as follows: 1- LPLUNC1, 2- Ezrin, 3- Actin, 4- Antileukoproteinase 1, 5- Chloride ion channel 1, 6- Complement C3, 7- PLUNC, 8- Dipeptidyl peptidase, 9- Polymeric-IG receptor, 10- Brain acid soluble protein, 11- Tubulin beta-chain, 12- CD9, 13- Gelsolin, 14- Superoxide dismutase, 15- Annexin A2, 16- EHD4, 17- Alpha-enolase,18- Tetraspanin-1, 19- EBP50 20- Complement factor B, 21- Calgizzarin, 22- Peroxiredoxin 1\&2, 23- ngALipocalin, 24- Calmyrin, 25- Aldehyde dehydrogenase, 26- Uteroglobin, 27- Pyruvate kinase isozymes M1/M2, 28- MUCIN-1, 29- Glutathione S-transferase P, 30- Cofilin-1, 31- Protein CGI-38, 32- Calcyphosine, 33- Ceruloplasmin, 34- Transgelin-2, 35- Calmodulin, 36- Stomatin, 37- Annexin A5, 38- Calpain-5,6, 39- Prominin 1, 40- Clusterin Apoliporotein J, 41- Cystatin-B, 42- Serpin F1, PEDF, 43- Protein S100 A6,8,9,14,16. 44- DMBT1, 45- Annexin A1, 46- CD59 glycoprotein, 47- Cathepsin D 48- Na/K ATPase alpha-1 chain, 49- Mucin-5B, 50- Prostate stem cell Ag 51- Galectin 9, 52- Leukocyte elastase inhibitor, 53- Galectin-3 BP, 54- Galectin 3, 55- WAP four-disulfide core protein2, 56- Defensin1, 57- Galectin 8, 58- Lysozyme C, 59- Serpins B3\&B6 60-MUCIN 4, 61-MUCIN-5AC, 62-MUCIN-16, 63- MUC-20.

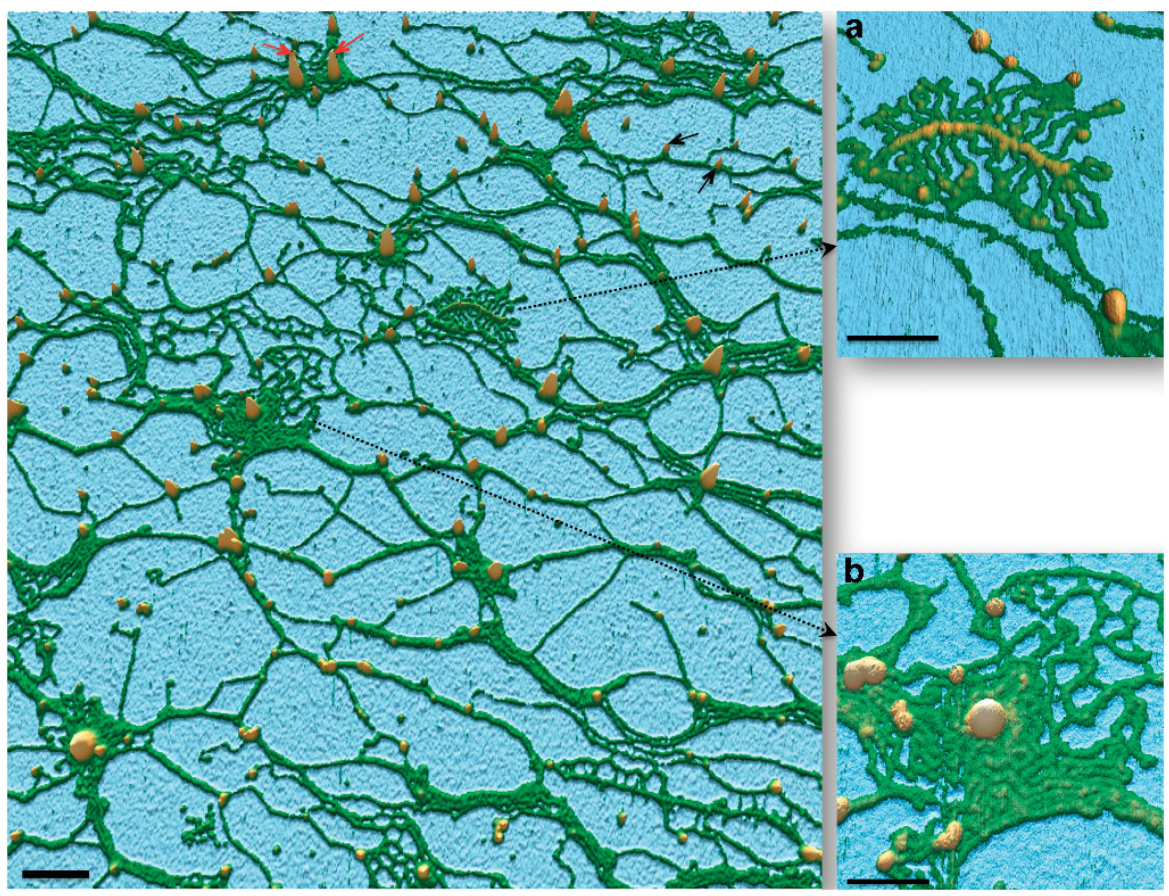

Figure 3 A representative 3-dimensional rendering of an Atomic Force Microscope (AFM) image of the organizational framework of the mucin interactome: the mucin interactome was isolated as described in the Methods and deposited onto mica and observed in a Cypher AFM. Glycosylated domains/chains of the mucins are shown in green and measured approximately $1-2 \mathrm{~nm}$ in height. The brown color represents protein regions of the framework, including mucin's naked protein domains, as well as small mucin-binding proteins, which range in height from 2 to $5 \mathrm{~nm}$ (black arrows, small nodes). Larger brown protein nodes with 5-12 nm heights (red arrows) in the framework indicate the presence of more than one, or larger, and/or more hydrophobic proteins on the nodes. (a) An oligomerized structure of a protein, possibly DMBT1, incorporated into the mucus network by interacting with mucin chains. (b) A compact/unfolded mucin form with a large globular node in the center. Scale bars: left $250 \mathrm{~nm}$, insets $100 \mathrm{~nm}$. 
spectrometry, and electron microscopy. Figure 4 summarizes the SEC-MALLS results. The figure shows the refractometry traces of the peak of the $\mathrm{V}_{0}$ region eluting from the $\mathrm{S} 1000$ column, which were obtained from the Optilab refractometer (Wyatt Technologies, Santa Barbara, CA) and overlaid with the molar masses of the eluting material, from MALLS. To determine the strength and stability of the mucin-protein complexes within an environment of increasing salt concentrations and ionic strength, the HBE secretions were subjected to increasing $\mathrm{GuHCl}$ concentrations ranging from 1 to $8 \mathrm{M}$. The effect of $\mathrm{GuHCl}$ on the recovery of the void complexes is shown in the titration experiment in Figure 4a. The first discernible decrease in the recovery of the void peak occurred at a $\mathrm{GuHCl}$ concentration of approximately $2 \mathrm{M}$ (green); the loss progressively increased through $4 \mathrm{~m} \mathrm{GuHCl}$ (magenta) and peaked at 6 and $8 \mathrm{~m} \mathrm{GuHCl}$ (light blue). The $8-\mathrm{m} \mathrm{GuHCl}$ treatment partially perturbed the interactome integrity, with more than half of the proteins observed in the residual complex (Figure 4b), indicating that $\mathrm{GuHCl}$ alone could not break all of the complex-forming interactions; notably, the complete disruption of the residual complexes required both $\mathrm{GuHCl}$

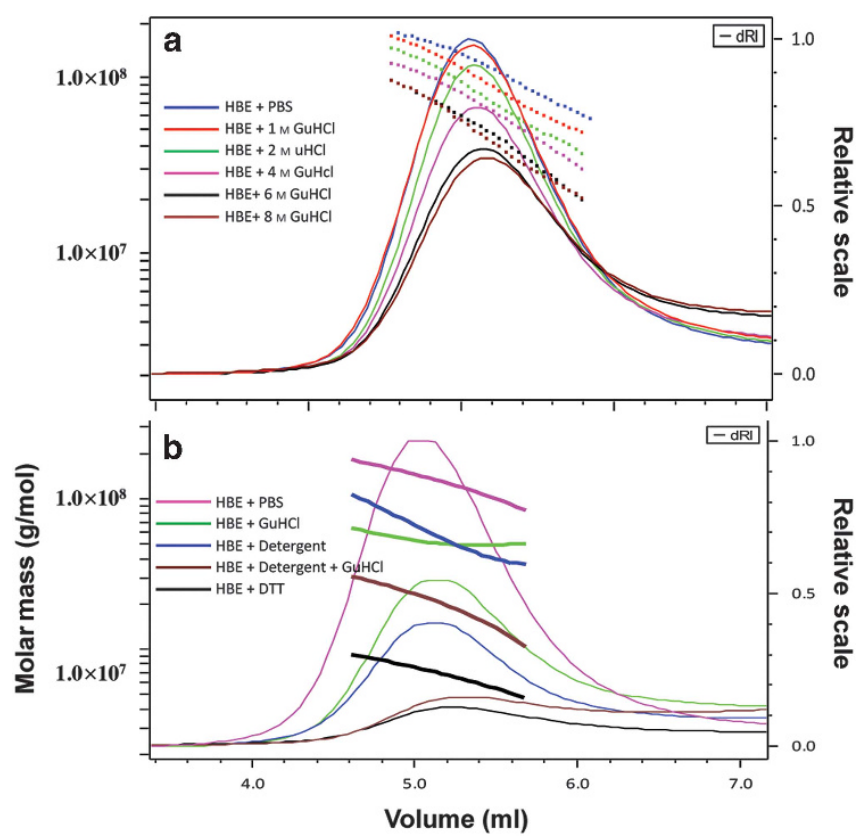

Figure 4 The effect of chaotropic agents on mucin interactome: (a) The effect of $\mathrm{GuHCl}(0-8 \mathrm{M})$ on the recovery of the void complexes: the results of the titration experiment show that the first discernible decrease in recovery of the void peak was observed at a $\mathrm{GuHCl}$ concentration of approximately $2 \mathrm{~m}$ (green; as indicated). The loss increases progressively through $4 \mathrm{~m} \mathrm{GuHCl}$ (magenta) and $6 \mathrm{~m}$ (black) to peak at $8 \mathrm{~m}$ (brown). (b) The effect of $\mathrm{GuHCl}$ and detergent on the recovery of the void complexes: the panel shows traces of the peak of the $V_{0}$ region from the $S 1000$ column, as obtained from the Optilab refractometer, and the molar masses, as obtained from Dawn multi-angle laser light scattering (MALLS), plotted across the mucin peak. The starting material, human tracheobronchial epithelial (HBE) cell-culture secretion (diluted in phosphate-buffered saline (PBS)), is denoted by the largest peak (magenta), and the subsequent dilution with $4 \mathrm{~m} \mathrm{GuHCl}$ (green) and $0.1 \% \mathrm{CHAPS} / 0.1 \%$ Triton is shown in blue. The results for $\mathrm{GuHCl}$ plus the detergent are shown in brown. and detergents (Figure $\mathbf{4 b}$ ). The starting material, HBE cell culture secretions (diluted in phosphate-buffered saline (PBS)), is represented by the largest peak (magenta; the apparent molecular masses of the native "S1000 complexes" were enormous, at approximately $133 \times 10^{6}( \pm 5 \%) \mathrm{g} \mathrm{mol}^{-1}$, and the calculated mass was $42.48 \mu \mathrm{g})$. Smaller peaks resulted from the addition of $4 \mathrm{~m} \mathrm{GuHCl}$ (green), 0.1\% CHAPS/0.1\% Triton (blue) and the combination of $\mathrm{GuHCl}$ plus the detergent (brown). Both $4 \mathrm{~m} \mathrm{GuHCl}$ and the detergents caused a substantial reduction in the molecular mass to approximately $60 \times 10^{6}( \pm 4 \%) \mathrm{g} \mathrm{mol}^{-1}$, and losses of material up to 24.09 and $16.69 \mu \mathrm{g}$, respectively. Treating the HBE secretions with both $\mathrm{GuHCl}$ and detergents caused an even more dramatic breakdown of the mucin-protein complexes: the apparent molar masses declined to $19.90 \times 10^{6}( \pm 2 \%)$ $\mathrm{g} \mathrm{mol}^{-1}$, and most of the material in the void disappeared, leaving only a residual $6.74 \mu \mathrm{g}$ mass (Figure 4). The molecular size of the HBE complexes (radius of gyration, $\mathrm{Rg}$ ) was compact in the PBS control at approximately $235 \mathrm{~nm}$ and was not substantially affected by the treatments (data not shown). The DTT treatment that cleaves mucins into monomeric units reduced the molecular weights to less than $4 \mathrm{~g} \mathrm{~mol}^{-1}$.

Electron microscopy analysis of the $S 1000$ void pool (i.e., the mucin interactome) of fresh HBE secretions isolated under "associative conditions" demonstrated the presence of abundant protein blobs reminiscent of globular proteins together with mucin chains and nodes ${ }^{15}$ in the complex (Figure 5). After the HBE secretions were treated with chaotropic agents, however, the number of protein blobs in the mucin structure was reduced (Figure 5). Typically, there are 15-20 blobs with an average size of $13 \mathrm{~nm}$ in the untreated complexes and 5-10 blobs in the treated samples. Figure $\mathbf{5}$ is also a typical example of the how the average shape $(\mathrm{Rg})$ was relatively consistent at approximately $200-230 \mathrm{~nm}$, even when the molecular weight was reduced dramatically from 150-200 MDa to 40-80 MDa.

\section{Monitoring functional consequences of individual mucin-protein interactions}

To assess the biological and biophysical effects of the mucinprotein interaction on the mucus microenvironment (hydration, viscosity, and elasticity), GST and galectin-3 were used to probe potential mucin-protein interactions. GST and galectin3 were selected because they were identified in the mucin interactome and available as purified proteins.

Characterizing the MUC5B-GST and MUC5B-galectin-3 interactions using quartz crystal mass balance methods (quartz crystal mass detection with dissipation). The binding of GST and galectin-3 to the preformed MUC5B layer was monitored with quartz crystal mass detection with dissipation (QCM-D). Figure 6 shows the frequency shift of overtone F7 (blue) and the dissipation shifts for the overtone D7 (red) after the mucin and proteins were exposed to the gold surface. Typically, after a 20min deposition of MUC5B on the gold surface, the $\Delta \mathrm{F} 7 / 7$ frequency decreases from -65 to -75 IE- 6 , while the $\Delta \mathrm{D} 7$ dissipation increases to 11.2 IE-6. Using the Sauerbrey model, 

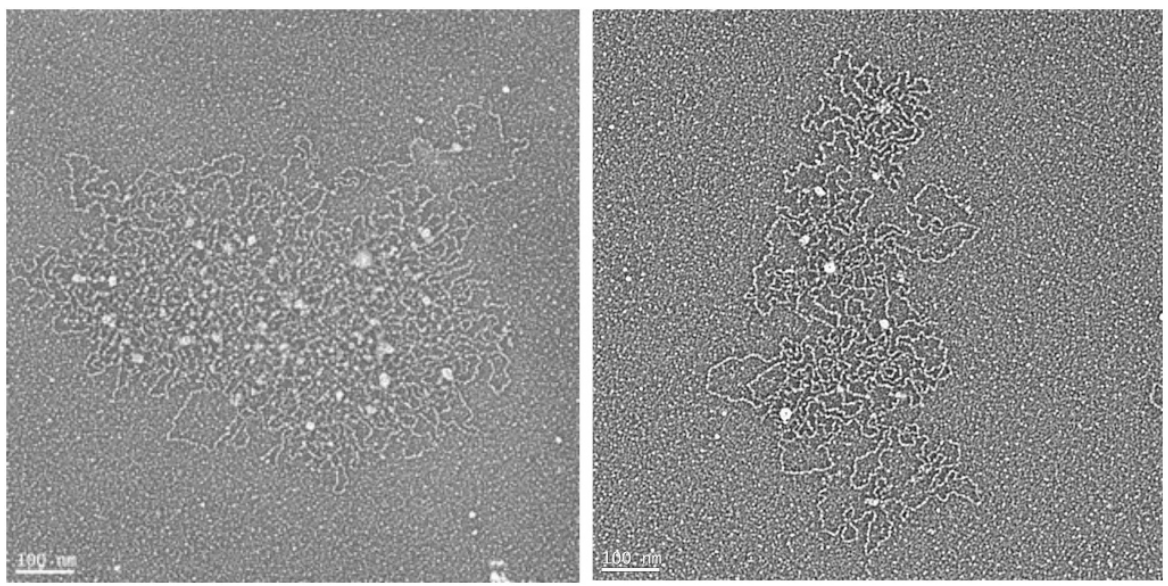

Figure 5 Organization of compact mucins in complex with other proteins under physiological and dissociative conditions, as observed with electron microscopy: a mucin-based complex isolated from human tracheobronchial epithelial (HBE) secretions under physiological conditions using S1000 chromatography. The compact form shown here contains abundant protein nodes on the mucin structure. The typical molecular weight of this structure is higher than 150-200 MDa. After the $\mathrm{GuHCl}$ + detergent treatment and S1000 isolation under dissociative conditions (eluted with $\mathrm{GuHCl}+\mathrm{CHAPS}$ ), the mucin structure was more relaxed, and quite a few protein nodes remained in the structure in a more organized manner. The molecular weight was dramatically reduced (to $40-100 \mathrm{MDa}$ ) after these treatments.

the quantity of the mucin bound to the surface was calculated to be $1,400 \pm 150 \mathrm{ng} \mathrm{cm}^{-2}$. The subsequent addition of bovine serum albumin (BSA) to block the naked gold surfaces shifted the $\Delta \mathrm{F}$ frequency to $-81 \mathrm{IE}-6$, which is equal to a mass deposition of $50 \pm 10 \mathrm{ng} \mathrm{cm}^{-2}$. The observation that this addition had no effect on the dissipation indicates that the albumin was only bound just to the naked gold surface and not to the mucin layer. After the addition of GST, however, the F7/7 frequency decreased to -87 IE- 6 , which is equal to an additional mass of $200 \pm 7 \mathrm{ng} \mathrm{cm}^{-2}$, and the D7 dissipation significantly increased to 12.14 IE-6. This change in dissipation suggests that GST had a substantial effect on the mucin layer, presumably by binding to mucins (Figure 6a). Voight modeling with the Q-tools software determined that the layer viscosity increased from $1.247 \mathrm{cP}( \pm$ s.e.m. $0.015, n=3)$ to $1.343 \mathrm{cP}$ ( \pm s.e.m. 0.017, $n=3)(P=0.005)$, and the shear elasticity increased from $9,783 \mathrm{~Pa}( \pm$ s.e.m. $460, n=3)$ to $12,510 \mathrm{~Pa}$ ( \pm s.e.m. $570, n=3) \mathrm{Pa}(P=0.015)$ after GST addition. In conjunction, the layer thickness increased from $32.07 \mathrm{~nm}$ ( \pm s.e.m. $1.18, n=3)$ to $32.87 \mathrm{~nm}$ ( \pm s.e.m. 1.01, $n=3$ ) $(P=0.043)$, which is an approximately $0.80-\mathrm{nm}$ increase.

After the addition of galectin-3 (Figure 6b), the F7/7 frequency decreased sharply to -90 IE-6, which is equal to an additional mass of $117 \pm 20 \mathrm{ng} \mathrm{cm}^{-2}$. During the course of galectin-3 binding (between minutes 22 and 30), the layer reorganized as shown by the drifting frequency and dissipation, suggesting that the binding was dynamic. After the final buffer wash, the system stabilized, and the $\Delta \mathrm{D} 7$ dissipation significantly decreased to 9.5 IE-6. Voight modeling with the Q-tools software determined that the layer viscosity increased from $1.180 \mathrm{cP}$ ( \pm s.e.m. $0.041, n=3)$ to $1.517 \mathrm{cP}( \pm$ s.e.m. $0.044, n=3)$ $(P=0.0052)$, and the shear elasticity increased from $7,336 \mathrm{~Pa}$ ( \pm s.e.m. $606, n=3)$ to $15,386 \mathrm{~Pa}( \pm$ s.e.m. $1,048, n=3)$ $(P=0.008)$ after the addition of galectin-3. In conjunction, the layer thickness decreased from $36.67 \mathrm{~nm}$ ( \pm s.e.m. 1.650, $n=3$ ) to $30.30 \mathrm{~nm}( \pm$ s.e.m. $1.652, n=3)(P=0.0025)$, which is an approximately $7 \mathrm{~nm}$ decrease in the layer thickness.

In QCM-D, an increase in dissipation or the linear $\Delta D / \Delta f$ ratio is associated with a highly hydrated layer with extended, flexible conformations. ${ }^{16}$ On the contrary, a decrease in dissipation and the $\Delta D / \Delta f$ ratio is associated with a dehydrated and/or stiffened layer. The combinational analysis of the frequency and dissipation change $(\Delta D / \Delta f)$ of mucin deposition and GST and Galectin-3 binding is presented in Figure 7. Mucins typically create a highly dissipated, well-hydrated layer, as indicated by the linear $\Delta D / \Delta f$ values in the figure. The addition of BSA changed the $\Delta D / \Delta f$ to a flat or a slight downward slope, suggesting the creation of a rigid layer on the surface. The subsequent GST addition changed the $\Delta D / \Delta f$ back to a linear upward trend, with a higher dissipation as the mass bound increased (Figure 7a). The addition of Galectin-3, however, first shifted the $\Delta D / \Delta f$ trend downward and then caused a sudden decline, which is consistent with crosslinking of mucins and a layer stiffening/collapsing effect (Figure $7 \mathbf{b}$ ).

Characterizing the nature of the MUC5B-GST interaction. To understand the nature and functional consequences of the MUC5B-GST interaction, we first added a known amount of GST protein $\left(100 \mu \mathrm{g} \mathrm{ml}^{-1}\right)$ to the MUC5B preparation of a known concentration $\left(100 \mu \mathrm{g} \mathrm{ml}^{-1}\right)$. We then isolated the complex using density-gradient centrifugation and measured the GST activity with and without mucin binding. A typical density-gradient profile (Figure 8a) shows that all of the MUC5B mucin was recovered in the high-density regions (fractions 7-11), whereas the majority of the GST was present in the low-density region (fractions 1-4). However, a significant percentage of the protein was observed in the MUC5B-rich fraction. The quantitative analysis indicated that approximately $20 \pm 5 \%$ of the spiked GST was detected in the mucin-rich pool (Figure 8a,b). 
a

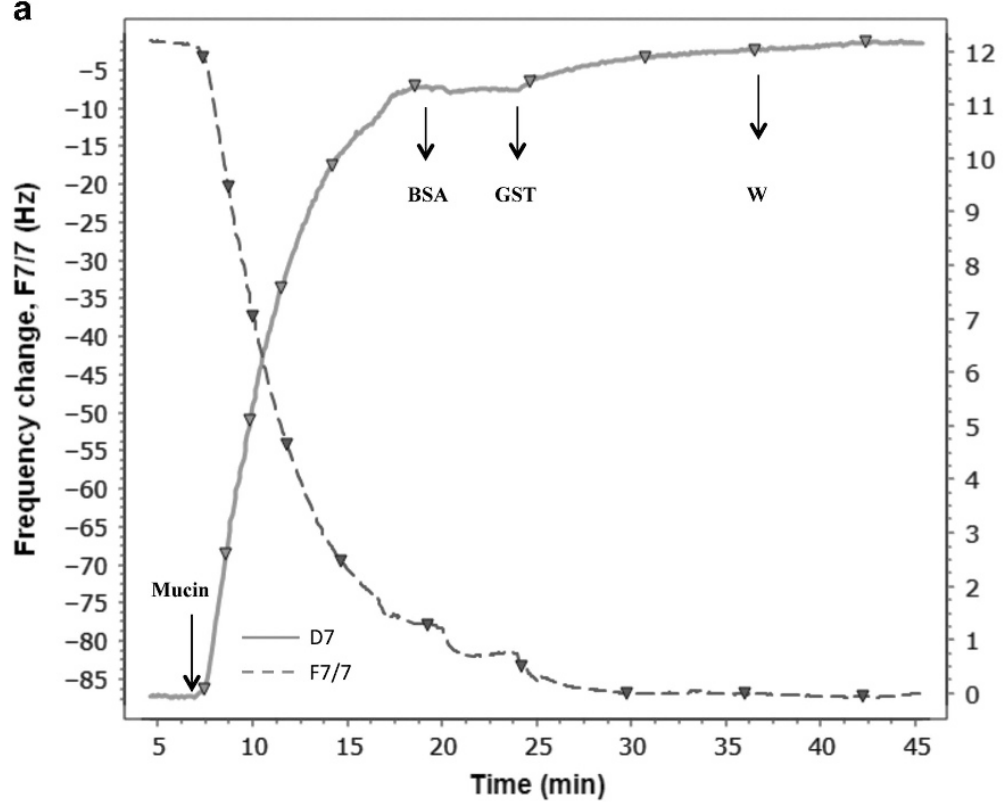

b

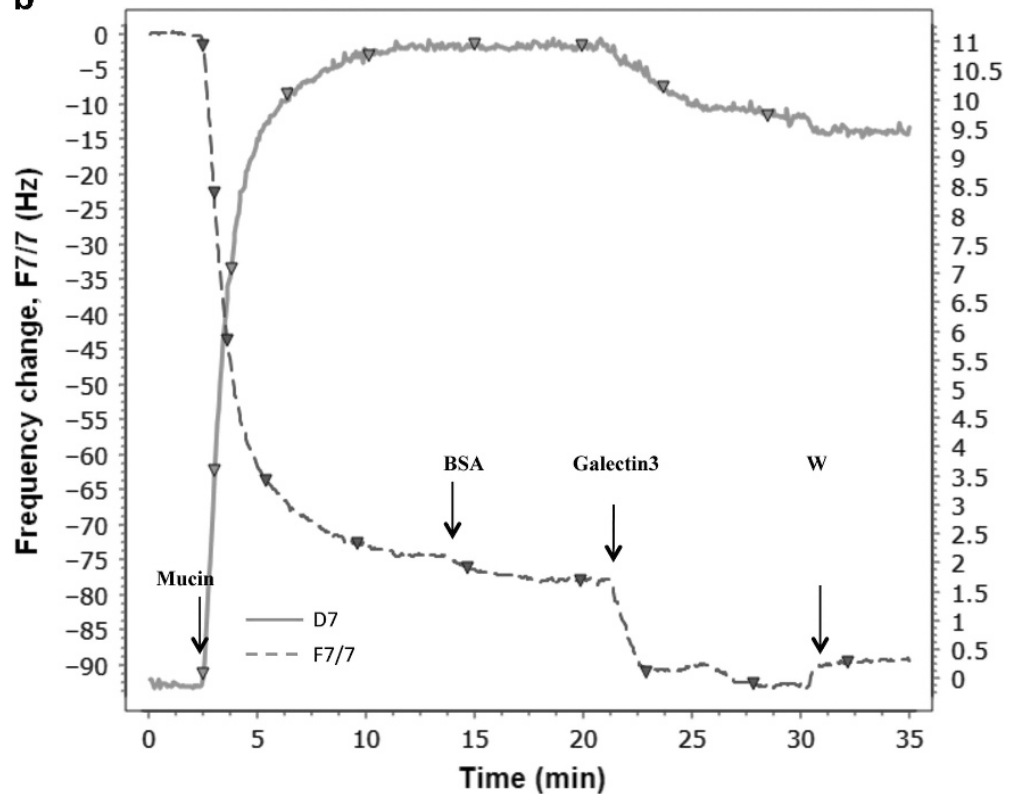

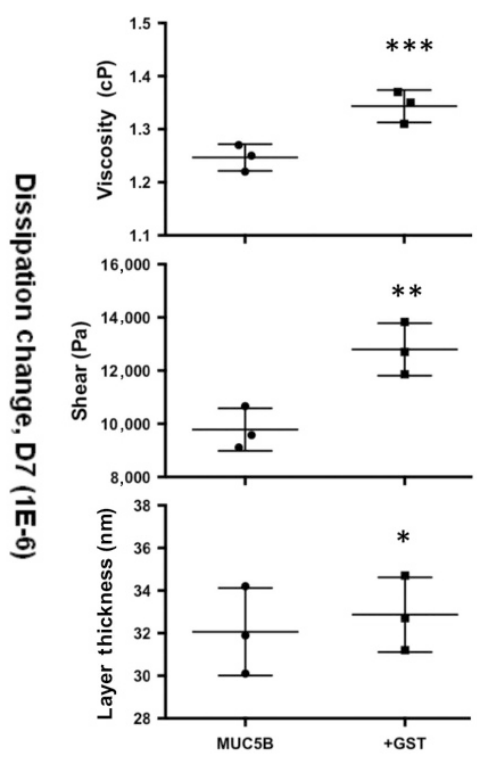

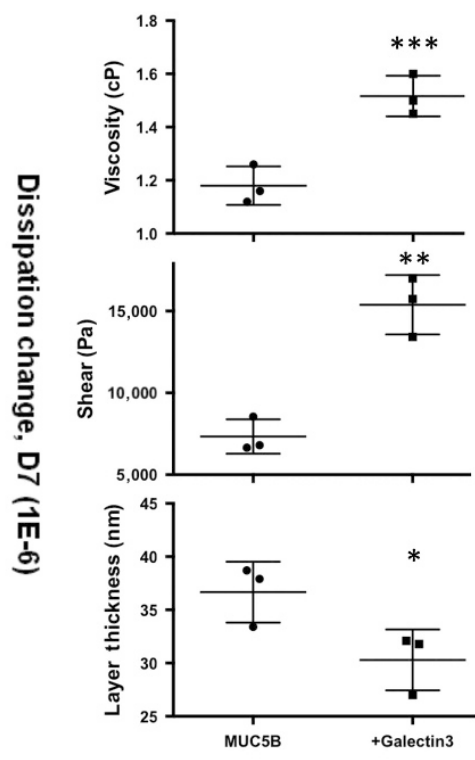

Figure 6 Monitoring individual mucin-protein interactions and their effects on the layer properties using quartz crystal mass detection with dissipation (QCM-D): the frequency shift one of the three of the overtones (F7, blue) and the dissipation shift for one of the three overtones (D7, red) were monitored using MUC5B + BSA + GST (a). The Sauerbrey model calculated an absorbed mucin layer of $1,600 \pm 5 \mathrm{ng} \mathrm{cm}{ }^{-2}$. The addition of BSA did not affect the properties of the layer. After the addition of GST, the frequency decreased to $\Delta \mathrm{F}=-87 \mathrm{IE}-6$, which was equal to an additional mass of $233 \pm 7 \mathrm{ng} \mathrm{cm}^{-2}$ and dissipation has significantly increased up to $\Delta \mathrm{D} 7=12,14 \mathrm{IE}-6$. The graphics in the right panel show the viscosity, shear and layer thickness comparisons before and after GST binding. The means and s.e.m. values are indicated by the major and minor horizontal bars, respectively. A paired samples $t$-test was used to determine changes in before/after values $\left({ }^{*} P=0.04,{ }^{* *} P=0.015\right.$, and $\left.{ }^{\star * \star} P=0.005\right)$. (b) Effect of galectin-3 on the mucin layer. The model calculated an absorbed mucin layer of $1,300 \pm 50 \mathrm{ng} \mathrm{cm}^{-2}$. Unlike GST, the addition of galectin-3 decreased the dissipation from 11.2 to 10.6 , suggesting that galectin-3 has a stiffening effect on the layer. There is a sharp decrease in the frequency and subsequent reorganization of the layer (broken arrow) from the galectin-3 addition until the buffer wash (W) at the end which significantly decreased the layer thickness. The graphics in the right panel show the viscosity, shear, and layer thickness comparisons before and after galectin-3 binding. The means and s.e.m. values are indicated by the major and minor horizontal bars, respectively. A paired samples $t$-test was used to determine the changes in the before and after values $\left({ }^{\star} P=0.0025\right.$, ${ }^{\star *} P=0.008$, and $\left.{ }^{\star * \star} P=0.005\right)$. BSA, bovine serum albumin; GST, glutathione S-transferase.

To investigate whether the interaction of GST with MUC5B affects the GST function, a GST assay was performed on the fractions from the $\mathrm{CsCl}$ density gradient. As shown in Figure 8c, GST activity peaked in the first 1-4 fractions (low-density region) and was dependent on the spiked amount (20 and $100 \mu \mathrm{g} \mathrm{ml}^{-1}$ ) of GST, but no measurable GST enzymatic activity was observed in the mucin-rich (8-10) fractions. The GST that was initially spiked in the mixture with 
a

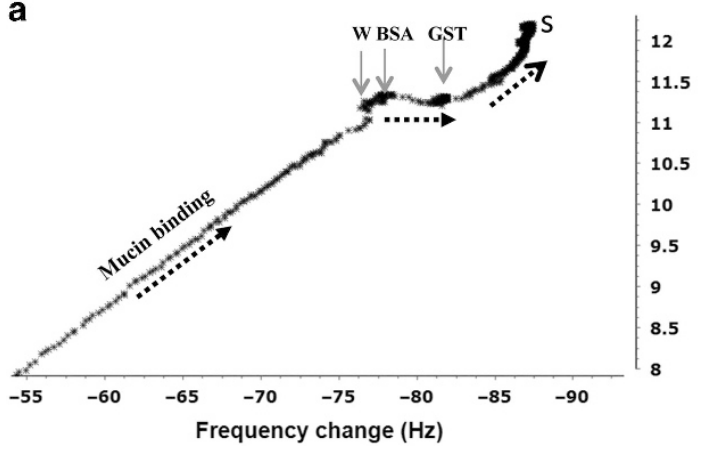

b

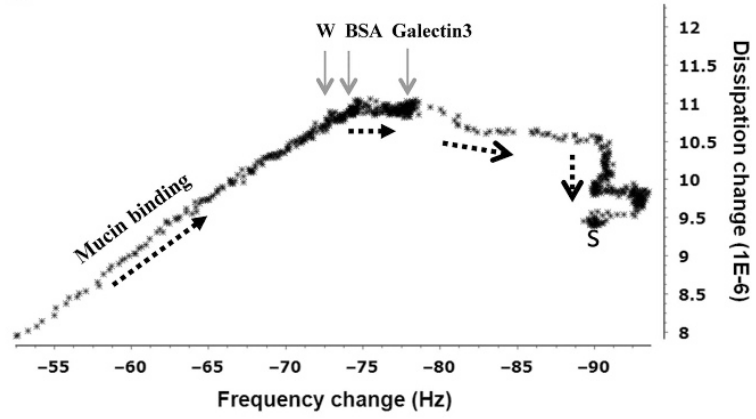

Figure 7 Frequency vs. dissipation changes, $\Delta D / \Delta f$, during the absorption of mucins, glutathione S-transferase (GST) and galectin-3. The linear $\Delta D / \Delta f$ corresponds to a highly hydrated layer (mucin binding). The addition of albumin changes the $\Delta D / \Delta f$ to a flat or downward slope, suggesting the formation of a rigid layer on the surface. The subsequent GST additions change the $\Delta D / \Delta f$ slope to a linear upward trend, with an increase in the mass bound resulting in greater dissipation (a). The addition of galectin-3 (b) first causes a slight and then sudden decrease in the dissipation and the $\Delta D / \Delta f$. The trend of the layer hydration/thickness is marked with broken arrows, and the stabilization of the layer at the end of the wash is marked (S).

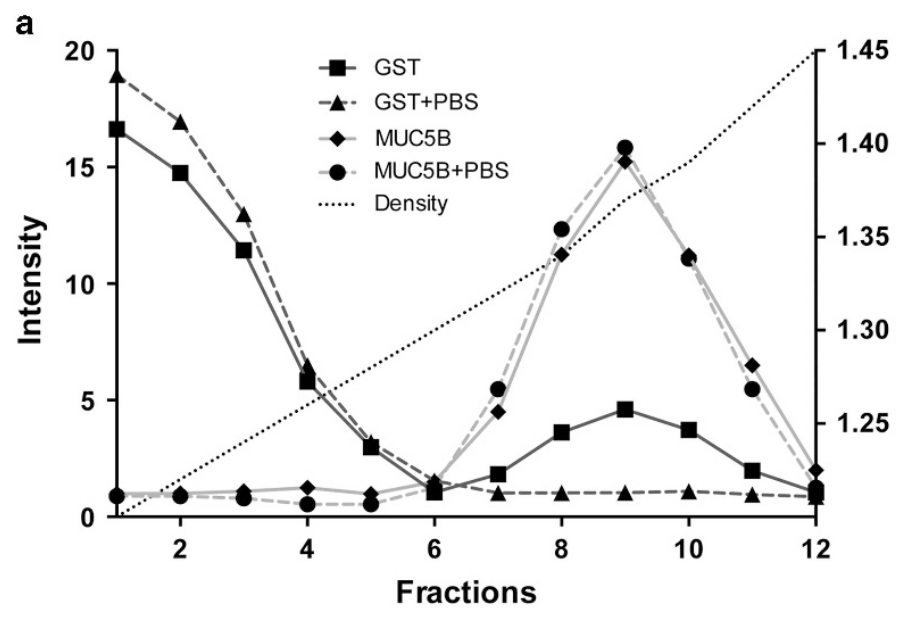

C

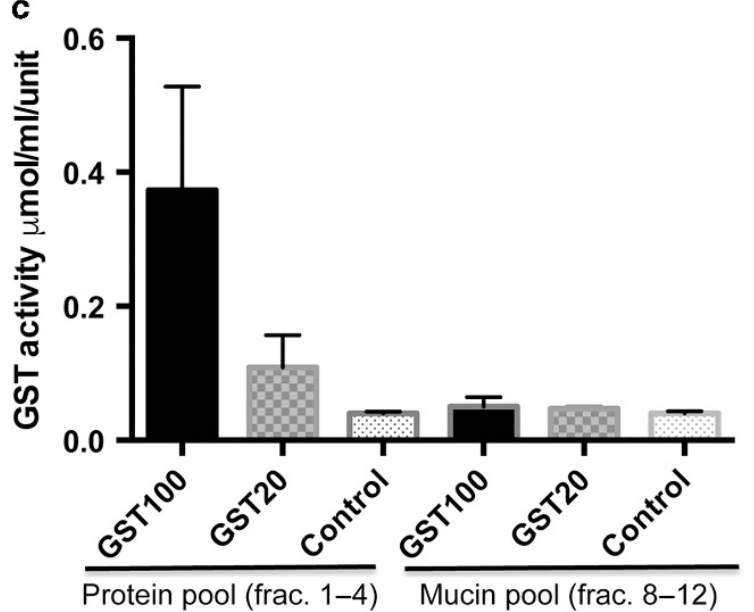

b

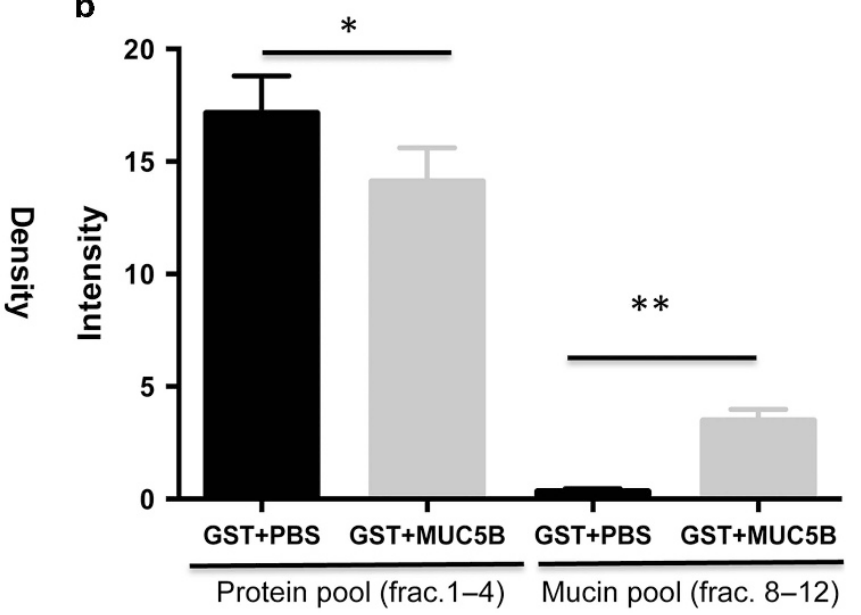

Figure 8 MUC5B-GST interaction: a known concentration of GST was used to spike the purified MUC5B preparation. Then, the complex was isolated using a cesium chloride $(\mathrm{CsCl})$ density gradient to detect the proportion of bound GST. A typical density-gradient profile (a) shows that all of the MUC5B mucin is recovered in the high-density regions (fractions 7-11), whereas approximately $15-20 \%$ of the GST was detected in the mucin region. CsCl density gradient with only GST (broken blue line) or MUC5B (broken magenta line) was used as controls. Four independent experiments using different preparations were summarized in (b) indicated that a significant amount of GST was present in the mucin fractions compared with the control $\left({ }^{*} P=0.002\right.$, $\left.{ }^{* *} P=0.0005\right)$. (c) the GST activity measurements over the gradient of different concentrations of GST (GST100 $\mu \mathrm{g}$, GST20 $\left.\mu \mathrm{g}\right)$ including a similar concentration in the mucin fraction at 7A $(\sim 15-20 \mu \mathrm{g})$ indicated that the GST activity peaks in approximately the first 3-4 fractions, but no measurable GST enzymatic activity was detected in the mucin-rich fractions (c). MUC5B preparation with no GST addition was used as a control. GST, glutathione S-transferase. 


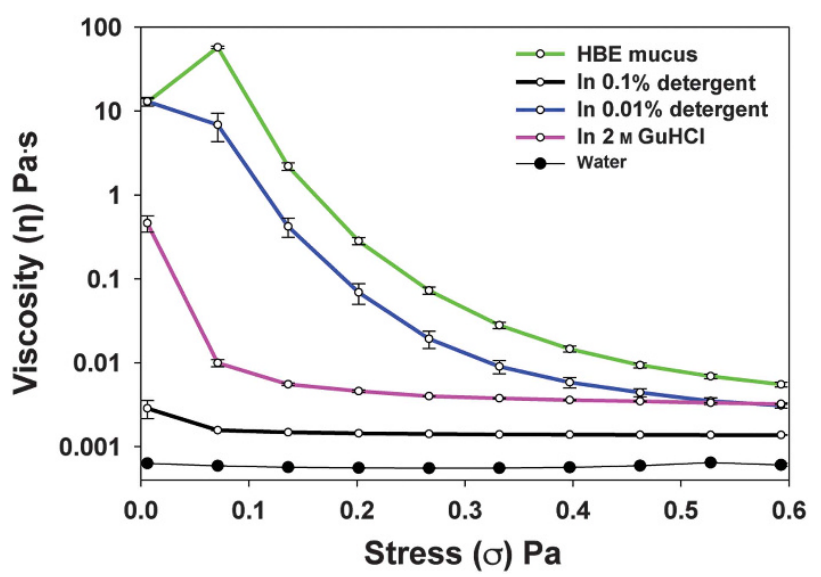

Figure 9 Rheological analysis of human tracheobronchial epithelial (HBE) secretions before and after the disruption of the protein-protein interactions: the HBE secretions were diluted so that all of the experiments had the same biomolecular concentration. The experiments were performed in a ramp-down manner starting at the highest shear stress and moving to the lowest. The data indicate that chaotropic agents and detergents significantly reduced the viscosity and abolished of the network viscoelasticity. GuHCl at $2 \mathrm{~m}$ caused a significant reduction in viscosity, whereas $0.1 \%$ detergent reduced the viscosity dramatically. The viscosity of water was also included (closed circles).

MUC5B had a final concentration of $100 \mu \mathrm{g} \mathrm{ml}^{-1}$. Because approximately $15-25 \%$ of GST is associated with MUC5B in the mucin-rich fractions, the concentration of GST should therefore be approximately $20 \mu \mathrm{g} \mathrm{ml}^{-1}$ which is far greater than the detection sensitivity (approximately $0.2 \mu \mathrm{g} \mathrm{ml}^{-1}$ of the GST functional assay. Additionally, the activity of an equivalent, $20 \mu \mathrm{g} \mathrm{ml}^{-1}$, concentration (GST20) of GST was measurable at the top of the gradient (Figure 8c).

\section{The effects of chaotropic agents and detergent on the bulk rheological properties of HBE cell secretions}

Rheological assessments of the HBE mucus were performed using a cone and plate rheometer with a $6-\mathrm{cm}, 4^{\circ}$-angle cone, which required an approximately a $1-\mathrm{ml}$ mucus sample. The output from a series of experiments is shown in Figure 9, where viscosity is plotted as a function of decreasing shear forces between 0.6 and $0.001 \mathrm{~Pa}$. The topmost data (green) indicate the dramatic shear force dependence of the viscosity of the native HBE mucus, with the viscosity varying by $4-5$ orders of magnitude. Below a shear force of $0.1 \mathrm{~Pa}$, the HBE sample acts essentially as an elastic solid, whereas at shear forces approaching $0.5 \mathrm{~Pa}$, the sample approaches the properties of water. The bottom data set shows the striking effect of adding $0.1 \%$ Triton to the sample; i.e., it completely abolished the sample's visco-elastic behavior. The data between these extremes show the effect of the presence of $0.01 \%$ Triton and $2 \mathrm{M} \mathrm{GuHCl}$. Clearly, a dramatic effect also occurred at levels of $2 \mathrm{M} \mathrm{GuHCl}$.

\section{DISCUSSION}

The available data on airway mucus suggest that there are significant gaps in our understanding of the relative contributions of the gel-forming mucins MUC5AC and MUC5B and the globular proteins to mucus gel function. Collectively, the data presented here strongly suggest that mucus must be viewed as a complex of mucins and proteins (Figures 1-3) and that globular proteins and non-gel-forming mucins significantly contribute to mucus gel pore size and viscoelasticity when they are linked to gel-forming mucins.

However, interactions between proteins and mucins do not necessarily indicate that all globular proteins modify mucus biophysically in a significant manner. In some instances, mucins may bind protective proteins that do not directly interfere with the mechanical properties of mucus but the interaction may contribute to the stability and/or function of globular proteins functional pathways. Supplementary Figure S3 shows putative pathways in which mucins and their interacting proteins might be involved. These functional networks indicate that mucins bind to proteins involved in anti-microbial (e.g., Defensin, LPUNC1, SPLUNC1, DMBT1, and complement 3), anti-oxidant (e.g., GST, peroxiredoxin, and superoxide dismutase) cell death/survival, cell signaling and molecular transport, (e.g., actin, gelsolin, S100 A6, A11, and transgellin), tissue development (e.g., annexin A1, calmodulin, CD9, CD59, and clusterin), fluid secretion (e.g., SPLUNC1, CLICP1), and inflammatory responses (e.g., Cofilin-1, CLIC1, ceruloplasmin, MUC1, MUC16, and alpha enolase). Although the mucin-binding proteins have a surprisingly large number of functionalities, e.g., antimicrobial, (anti) protease, antioxidant and wound healing, they can be organized into a large category; innate host defense.

The structural multi-domain properties of mucin macromolecules modulate the nature of these interactions. Mucin macromolecules are dominated by charged oligosaccharides, raising the possibility that electrostatic interactions contribute to mucin protein-binding characteristics. Our data suggest that proteins, such as galectins $-3,-8$ and -9 , bind to mucins through relatively weak electrostatic interactions, e.g., they can be freed from mucins using solvents with high ionic strength. Mucins also have un-glycosylated protein regions at their $\mathrm{N}$ - and/or $C$-terminal regions, which contains hydrophobic regions. ${ }^{17}$ Our light scattering data (Figure 4) suggest that the majority of the proteins interact with mucins via electrostatic and/or weak hydrophobic interactions, whereas some proteins interact through very strong hydrophobic and/or covalent interactions. For instance, the proteomic analysis of samples treated with $\mathrm{GuHCl}$ and detergent treatments indicated that proteins such as LPLUNC1, GST, and complement factor C3 remained bound to mucins after harsh treatments. The electron microscopy analysis of the isolated complexes (Figure 5) also suggests that mucin complexes not only lose proteins after detergent and/or GuHCl treatments, but the mucin macromolecule chains also lose the structure. These data suggest that globular proteins have a role in maintaining their highly oligomeric structures and defining the hydrodynamic volume and molecular weight of mucins in solution.

In addition to mucin-protein interactions, we show here there are also mucin-mucin interactions that may cause clustering of the linear mucin chains, which may affect the 
barrier properties of the mucus gel (Figure 3). It has been shown that associations between large polymer chains can have a major effect on the viscoelasticity of the networks, ${ }^{18,19}$ and these networks can be modified by groups that connect the macromolecules to each other. ${ }^{20}$ Electrostatic interactions may also cause crosslink mucin polymers and change the biophysical properties of the mucus gel. ${ }^{21}$ We have previously shown electron microscopic evidence ${ }^{15}$ that demonstrates associations within and between the $N$ - and $C$ - terminal domains that organize the intra- and inter-molecular properties of the salivary MUC5B mucin and contribute to its specific macromolecular architecture. The studies on saliva and isolated MUC5 $\mathrm{B}^{18}$ and the recombinant version of MUC5B $N$-terminal region $^{22}$ suggest that the protein domains of the mucins themselves may undergo weak (but specific) and sometimes strong calcium-mediated interactions. In addition, hydrophobic interactions ${ }^{23}$ are also important for mucin entanglement and crosslinking, and, therefore determine the properties of the mucus gel. ${ }^{21,24}$ Taken together, mucin-mucin interactions are also major determinants of the properties of the resulting mucus gel including pore size and viscoelasticity.

To date, no studies have demonstrated the effects of the globular proteins that interact with the gel-forming mucins via electrostatic, hydrophobic, or covalent interactions on the biophysical property of the mucus gel. Our data shed some light on the functional consequences of such interactions. Hundreds of globular proteins contribute to the biological complexity of mucus; ${ }^{8,9}$ however, apparently only a handful of proteins modulate its rheological properties. Here, we have shown that 6 major mucins and approximately 30 globular proteins are present in complexes in HBE cell culture secretions (Figure 2). To investigate the consequences of such interactions, GST (which likely interacts with the mucin's naked protein regions) MUC5B and galectin-3 (which interacts with the mucin's carbohydrate region) MUC5B interactions were examined in this study. We have previously used the QCM-D technique to investigate the biophysical properties of mucin layers. ${ }^{25}$ Here, we used this sensitive technique to assess the visco-elastic properties of thin mucin films and to measure response to exposure purified proteins.

GST is one of the most abundantly secreted proteins in the mucus gel and has an important role in mucosal health by maintaining oxidative balance. ${ }^{26}$ Galectins (galactose-binding proteins) are relatively well studied in terms of their interaction with membrane-tethered mucins and their contribution to mucosal defense. ${ }^{27-29}$ Interactions of these proteins with membrane mucins, consequently, may promote immune modulation and innate protection. ${ }^{30,31}$ However, their interactions with gel-forming mucins and their contribution to the visco-elastic properties of mucus are not well studied. Our QCM-D data suggest that GST binding increased the dissipation of mucin molecules to, reflecting an upwards expansion from the surface and producing significant changes on the hydration, viscosity, and elasticity of the layer (Figures 6a and $7 \mathbf{a}$ ). Galectin-3 addition, in contrast, caused a dramatic decrease in dissipation, coupled to significant increases in layer viscosity and the elasticity, and decreased thickness of the layer (Figures $\mathbf{6 b}$ and $\mathbf{7 b}$ ). These data strongly suggest that galectin-3 had a substantial stiffening effect on the layer by binding to mucins and, most likely, crosslinking them. Taken together, the addition of the GST and Galectin-3 to an already formed mucin layer had a substantial adverse effect on the hydration and visco-elastic properties of the layer. This result suggests that the interactions via mucin's protein (GST) and carbohydrate regions (galectin-3) may have different consequences, and the hydration and the biophysical properties of the mucus layer can accordingly be dynamically tuned by these interactions.

To understand whether these interactions affect the "primary" function of the binding globular proteins, we performed an enzyme activity experiment using the MUC5B-GST interaction as a model. The interaction affected the micro-rheology of the mucin layer (Figures 6 and 7) and could also simultaneously regulate its biological activity (Figure 8) as we did not observe biological GST activity in the mucin-rich fractions even though approximately 15-20 $\mu \mathrm{g}$ of GST was present in the fractions. This result suggests that mucin may have a functional modulatory role in regulating the function of bound proteins. However, this effect cannot be generalized to all interactions. Along with the same line, it was previously suggested that salivary MUC7, a small secreted nongel-forming mucin, interacts with salivary amylase and enhances its activity. ${ }^{32}$

Glandular proteins, such as TFF (Trefoil factor) peptides ${ }^{33,34}$ and IgGFc-binding proteins (FCGBP), ${ }^{35,36}$ are mucin-interacting proteins that may also change the mucus properties. When they are over-secreted and hyper-concentrated, together with mucins, in the mucosal surfaces, they cause the formation of a non-transportable static mucus ${ }^{37}$ which may become a site for infection and inflammation. Analyses of human lung secretions, such as bronco-alveolar lavage and sputum, have detected these proteins present in the mucin interactome (unpublished data). We did not detect significant amount TFF peptides or FCGBP in HBE mucus, likely because the HBE cell culture that we used primarily had a surface epithelial phenotype rather than a glandular phenotype. This result may indicate that TFF and FCGBP are mostly products of glandular cells or are expressed at very low levels under sterile culture conditions. However, after the HBE cell cultures were challenged with Pseudomonas aeruginosa and the supernatant of mucopurulent material from cystic fibrosis lungs, increased concentrations of these two proteins were detected in the washings. ${ }^{38}$

The mechanics, dynamics, and biochemical and biophysical stability of mucin networks formed through mucin-mucin and mucin-protein interactions were assessed with chaotropic agents and detergents, which are known to disrupt noncovalent protein interactions. Specific protein-protein and protein-carbohydrate interactions may be modified in a number of ways. If these interactions are electrostatic in nature, then they may be abolished by ionic strength; if they are hydrophobic, then detergents (such as CHAPS and Triton) and 
chaotropes (such as urea and guanidinium hydrochloride) should significantly interfere with the interactions. The reversibility vs. irreversibility of these procedures also yields insight into the presence and nature of the crosslinking mechanisms that contribute to the gel. As outlined in Figures 4 and 5, HBE secretions appear to be composed of complex associations between mucins and proteins, which can be disrupted by chaotropic agents and detergents. The rheology data (Figure 9) show dramatic changes in the shear forcedependence viscosity of the HBE mucus when treated with detergents and chaotropic agents, suggesting that the mucinprotein interactions constitute a major component of mucus rheological properties.

Based on our data and previous studies, we speculate that the mucin-protein interactions have several functional consequences: (i) These interactions may be an important parameter for the intra-granular packaging, unpackaging of the mucin macromolecules following granular release, and their subsequent unfolding/maturation dynamics; (ii) The interactions affect the visco-elastic properties of the layer and therefore optimize mucociliary clearance and increase the stability of the barrier function; (iii) The binding proteins protect (or sometimes facilitate) the proteolysis of the naked protein regions of mucins; and (iv) Highly glycosylated mucins may protect the interacting proteins from proteolytic attacks, concentrate them in regions of action, and extend their lifetime with respect to important biological functions on airway surfaces. These findings need to be further explored in other experimental systems, including disease models, in which the composition of the interactome may be changed and their contribution to the pathogenesis of chronic lung diseases, such as cystic fibrosis and chronic obstructive pulmonary disease, assessed.

In conclusion, we propose that the visco-elastic properties of functioning airway mucus do not simply depend on the biophysical properties of the mucins themselves but also on the dynamic and/or stable interactions between mucins and globular proteins to form an optimally functioning mucus gel for effective biological and mechanical innate immune protection. These observations challenge the paradigm that mucins alone confer most of the rheological properties of mucus and will ultimately lead to a greater understanding of the function of this complex system. This information will be helpful for effectively treating mucus pathologies with better therapeutic applications that specifically or generically optimize these interactions.

\section{METHODS}

Cell culture and mucus collection. HBE cells were obtained from the resected airways of normal donor tissue from the University of North Carolina using Institutional Review Board-approved protocols. Primary airway epithelial cells from three normal donors with no history of lung disease were isolated and expanded on plastic to generate passage- 1 cells, which were then plated at a density of $6 \times 10^{5}$ cells per well on permeable 24-mm-diameter supports (T-Clears, Transwell). ${ }^{39}$ After air-liquid interface culturing for 4 weeks, the cells formed well-differentiated, polarized HBE cultures that resembled the pseudostratified mucociliary epithelium in vivo. ${ }^{40}$ We obtained the mucous secretions by incubating $1 \mathrm{ml}$ of PBS on the apical surface of the cultures for $30 \mathrm{~min}$ at $37^{\circ} \mathrm{C}$, removing the PBS with a large-caliber pipette, and repeating the procedure, thus obtaining $2 \mathrm{ml}$ per wash per culture. The washes were obtained from four biological replicate cultures from three different donors and then pooled for each donor. Each sample was immediately placed on ice, and subsequently centrifuged at $300 \mathrm{~g}$ for $10 \mathrm{~min}$ to remove the cells. The samples were subjected to gel filtration and subsequent isopycnic centrifugation (see below).

\section{Gel filtration and density-gradient isolation of mucin complexes.}

Mucins and their interacting proteins were isolated using a two-step procedure involving size-exclusion chromatography fractionation followed by density-gradient fractionation. The HBE secretions were centrifuged at 2,000 $g_{\text {av }}$ for $10 \mathrm{~min}$ at $4{ }^{\circ} \mathrm{C}$. The supernatant was decanted and stored at $4{ }^{\circ} \mathrm{C}$ and then subjected to gel permeation chromatography on a Sephacryl-1000 (S-1000, $50 \times 2.5 \mathrm{~cm})$ eluted with $200 \mathrm{~mm} \mathrm{NaCl}$ containing $10 \mathrm{~mm}$ EDTA, $\mathrm{pH} 7$, at a flow rate of

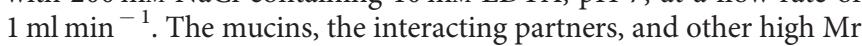
protein complexes that were eluted in the void volume of the column were then subjected to $\mathrm{CsCl}$ density-gradient centrifugation at a starting density of $1.45 \mathrm{~g} \mathrm{ml}^{-1}$ in a Beckman Ti45 angle rotor at 40,000 r.p.m. for $60 \mathrm{~h}$ at $14{ }^{\circ} \mathrm{C}$. The samples were unloaded from the top, and 2-ml fractions were collected and subjected to PAS and alcian blue staining and mucin immunoblotting using slot blot analyses, as previously described. The pooled fractions from the top (fractions 1-6), middle (fractions 8-15, mucin MUC5AC and MUC5B rich), and bottom (fractions 16-20, keratan sulfate rich) were dialyzed into $200 \mathrm{~mm} \mathrm{NaCl}$ containing $10 \mathrm{~mm}$ EDTA at pH 7.0 for further analysis.

Mass spectrometry. Mass spectrometry analysis of the isolated complexes was essentially performed as previously described. ${ }^{8,17,41}$ The pools from the gel filtration chromatography and density-gradient centrifugation were reduced, alkylated, and digested using trypsin. The peptides were separated from large glycopeptides using Sephacryl 200 (S200) size-exclusion chromatography. ${ }^{41}$ The S200 peptide pool from was subjected to nano-LC-MS/MS (liquid chromatography-tandem mass spectrometry) analysis. All data were acquired using a Waters Q-Tof micro hybrid quadrupole orthogonal acceleration time-of-flight mass spectrometer (Waters, Manchester, UK) and the manufacturer's MassLynx 4.0 software. The processed data were searched against an updated NCBInr and Swiss-Prot databases using the Mascot search engine.

SEC-MALLS analysis of mucin complexes. The HBE mucus samples were diluted 1:1 in PBS or the chaotropic agent or detergents and subjected to chromatography on a Superdex 1000 size exclusion column $(15 \times 2.5 \mathrm{~cm})$ and eluted with $0.2 \mathrm{M} \mathrm{NaCl}$ (with $10 \mathrm{~mm}$ EDTA) at a flow rate of $500 \mu \mathrm{lmin}^{-1}$. The column effluent was passed through an in-line enhanced optimal system laser photometer (Dawn, Wyatt Technologies) coupled to a digital signal-processing interferometric refractometer (Wyatt/Optilab) to continuously measure the light scattering and sample concentrations, respectively. The captured data were integrated and analyzed using the Astra software (Wyatt Technologies) provided with the Dawn laser photometer (Wyatt Technologies).

Measuring mucus viscosity. The complex viscosity of the HBE mucus samples was determined based on the shear dependence of the viscosity using a Bohlin Gemini Rheometer (Malvern Instruments, Worcestershire, UK), with a $20-\mathrm{mm}$ diameter parallel plate set at a gap thickness of $50 \mu \mathrm{m}$. The HBE mucus diluted in the treatment buffers containing $0.01-0.1 \%$ Triton X-100, and $2 \mathrm{M} \mathrm{GuHCl}$ or in PBS such a way that all experiments are at the same concentrations of biomolecules. All experiments were performed over a stress range of $0.001-0.6 \mathrm{~Pa}$ and at a frequency of $1 \mathrm{~Hz}$ (intermediate frequency between those associated with tidal breathing and mucociliary clearance). All analyses were performed at $23^{\circ} \mathrm{C}$ to minimize sample 
dehydration. The data were analyzed using the software provided with the instrument.

Quartz crystal mass detection with dissipation. The structural properties of the absorbed mucins and their interacting proteins were measured on quartz crystals using the quartz crystal microbalance with dissipation monitoring (QCM-D; Q-sense, Sweden). QCM-D offers a micro-mechanical method for dynamically investigating the relationship between complexes and proteins and determining which proteins bind to them. By first binding purified mucins to the goldcoated chip and subsequently adding purified protein(s), it is possible to determine both the amount of protein that binds to the mucins (based on a change in frequency) and the effect of this binding on the local viscoelasticity and hydration of the mucin network (based on changes in dissipation). We successfully applied this unique method to measure the functions of the gel-forming MUC5B mucin through the dissipative properties of its absorbed layers on the gold-coated crystal. $^{25}$ This study used sensor crystals coated with pure gold. All protein and mucin solutions were dissolved in a $200-\mathrm{mm} \mathrm{NaCl}$ solution with $10 \mathrm{~mm}$ EDTA using high-purity Milli-Q water. The buffer was filtered through 0.2 -micron filters and degassed via sonication before use. Approximately $100 \mu \mathrm{g} \mathrm{ml}^{-1}$ preparations of MUC5B (isolated from saliva as previously described ${ }^{25,42}$ ), GST (GST-P, Sigma-Aldrich, St Louis, MO) and galectin-3 (Sigma-Aldrich) BSA (Sigma-Aldrich) were used for the QCM-D binding experiments. As is typically performed, MUC5B was deposited until it reached a saturation level. Albumin was then applied to the layer to block any possible naked regions on the gold chip. Next, the target molecule was applied to the mucin layer, and buffer washes were performed between each addition. All experiments were conducted at a constant temperature of $26^{\circ} \mathrm{C}$. the solutions were flowed over the crystal at $50-100 \mu \mathrm{min}^{-1}$ at concentrations that typically ranged between 50 and $100 \mu \mathrm{g} \mathrm{ml}^{-1}$. The changes in the frequency (mass deposition) and dissipation (rigidity, hydration of the layer) of the crystal were measured by recording the response of a freely oscillating crystal that had been vibrated at its resonance frequency with its fundamental frequency and overtones (e.g., 15, 25, and $35 \mathrm{MHz}$ ). The quantity of the bound molecules was calculated by applying the Sauerbrey model. ${ }^{25}$ The effects of the binding on layer viscosity, elasticity (shear modulus), and thickness were calculated by applying the Voight visco-elastic model and the Q-Tools provided by the manufacturer.

GST and mucin interaction measurements. For the GST and mucin interaction studies, $20-100 \mu \mathrm{g} \mathrm{ml}^{-1}$ human GST (GST in PBS, isolated from human placenta, Sigma-Aldrich) and $100 \mu \mathrm{g} \mathrm{ml}^{-1}$ MUC5B mucin (in PBS, isolated from saliva) were mixed and incubated for $10 \mathrm{~min}$ at room temperature. The mixture was then subjected to $\mathrm{CsCl}$ density-gradient centrifugation to separate the mucin from the protein. The samples were adjusted to a density of $1.35 \mathrm{~g} \mathrm{ml}^{-1}$ by the addition of solid $\mathrm{CsCl}$. The samples then were centrifuged in a $70.1 \mathrm{Ti}$ rotor at 50,000 r.p.m. in a Beckman L8-M ultracentrifuge (Beckman Coulter, Indianapolis, IN) for $65 \mathrm{~h}$ at $14^{\circ} \mathrm{C}$. Then, the centrifugation fractions were unloaded from the top and analyzed for GST (GSTP1 polyclonal antibody, Sigma-Aldrich) and MUC5B (MUC5BIII antibody) immunoreactivity using slot blotting. The fractions were also subjected to the GST activity analysis using a GST assay kit (Sigma-Aldrich), according to the manufacturer's instructions. $\mathrm{CsCl}$ density gradients with only GST and MUC5B were used as controls.

Isolation of mucin complexes for AFM imaging. For AFM imaging purposes, the mucin interactome was isolated using a specific protocol to preserve membrane-tethered mucins in the network while removing vesicles/exosomes from the sample, which would interfere with the imaging. HBE secretions were ultracentrifuged for $1 \mathrm{~h}$ at 19,000 r.p.m. in an L8-70 (Beckman Coulter) using a swing out rotor (SW40ti) to remove vesicles. The supernatant was collected and layered onto a 1-ml cushion of $\mathrm{CsCl}\left(1.35 \mathrm{~g} \mathrm{~cm}^{-3}\right)$ in PBS. Samples were then ultracentrifuged at 40,000 r.p.m. for $2 \mathrm{~h}$ and the mucin-rich material was collected from the cushion and then run through a Sepharose CL2B gel filtration column using PBS as the elution buffer. The mucins and their interacting proteins were recovered in the void volume for further AFM imaging. For Supplementary Figure S1, a dissociatively purified MUC5B preparation (from saliva as previously described ${ }^{25,42}$ ) was used.

Fifty microliters of a 10-mm Nickel Chloride solution was placed onto a freshly cleaved mica surface. After $1 \mathrm{~min}$, the excess $\mathrm{NiCl}_{2}$ was rinsed off with deionized water and a $60-\mu$ ldroplet of the sample was pipetted onto the surface. The sample was allowed to deposit onto the surface for $3 \mathrm{~min}$, after which it was rinsed with deionized water and blown dry with nitrogen. Imaging took place on a Cypher AFM system (Asylum Research, Santa Barbara, CA) using an ARROW-UHF AuD20 cantilevers (NanoWorld Innovative Technologies, Neuchatel, Switzerland) operating in non-contact mode. The raw images were processed using the software provided by the manufacturer.

Statistical analysis. Where applicable, the statistical analyses were performed using the paired samples $t$-test.

SUPPLEMENTARY MATERIAL is linked to the online version of the paper at http://www.nature.com/mi

\section{ACKNOWLEDGMENTS}

We thank the late Dr Sheehan for his inspiration. We also thank Drs Boucher and Davis for critically reading the manuscript. This study was supported by a grant from the National Institutes of Health R01HL103940.

\section{DISCLOSURE}

The authors declared no conflict of interest.

Official journal of the Society for Mucosal Immunology

\section{REFERENCES}

1. Kesimer, M., Ehre, C., Burns, K.A., Davis, C.W., Sheehan, J.K. \& Pickles, R.J. Molecular organization of the mucins and glycocalyx underlying mucus transport over mucosal surfaces of the airways. Mucosal Immunol. 6, 379-392 (2013)

2. Sheehan, J.K., Kesimer, M. \& Pickles, R. Innate immunity and mucus structure and function. Novartis Found. Symp. 279, 155-166 (2006). discussion 167-169, 216-269.

3. Rubin, B.K. Physiology of airway mucus clearance. Respir. Care 47, 761-768 (2002).

4. Rogers, D.F. Mucus hypersecretion in chronic obstructive pulmonary disease. Novartis Found. Symp. 234, 65-77 (2001). discussion 77-83.

5. Rubin, B.K. Mucus structure and properties in cystic fibrosis. Paediatr. Respir. Rev. 8, 4-7 (2007).

6. Sheehan, J.K., Richardson, P.S., Fung, D.C., Howard, M. \& Thornton, D.J. Analysis of respiratory mucus glycoproteins in asthma: a detailed study from a patient who died in status asthmaticus. Am. J. Respir. Cell Mol. Biol. 13, 748-756 (1995).

7. Ali, M., Lillehoj, E.P., Park, Y., Kyo, Y. \& Kim, K.C. Analysis of the proteome of human airway epithelial secretions. Proteome Sci. 9, 4 (2011).

8. Kesimer, M. et al. Tracheobronchial air-liquid interface cell culture: a model for innate mucosal defense of the upper airways? Am. J. Physiol. Lung Cell. Mol. Physiol. 296, L92-L100 (2009).

9. Nicholas, B. et al. Shotgun proteomic analysis of human-induced sputum. Proteomics 6, 4390-4401 (2006).

10. Button, B. et al. A periciliary brush promotes the lung health by separating the mucus layer from airway epithelia. Science 337, 937-941 (2012).

11. Thornton, D.J., Rousseau, K. \& McGuckin, M.A. Structure and function of the polymeric mucins in airways mucus. Annu. Rev. Physiol. 70, 459-486 (2008).

12. Thornton, D.J., Carlstedt, I., Howard, M., Devine, P.L., Price, M.R. \& Sheehan, J.K. Respiratory mucins: identification of core proteins and glycoforms. Biochem. J. 316 (Pt 3), 967-975 (1996).

13. Kesimer, M. et al. Characterization of exosome-like vesicles released from human tracheobronchial ciliated epithelium: a possible role in innate defense. FASEB J. 23, 1858-1868 (2009). 
14. Madsen, J., Mollenhauer, J. \& Holmskov, U. Review: Gp-340/DMBT1 in mucosal innate immunity. Innate Immun. 16, 160-167 (2010).

15. Kesimer, M., Makhov, A.M., Griffith, J.D., Verdugo, P. \& Sheehan, J.K. Unpacking a gel-forming mucin: a view of MUC5B organization after granular release. Am. J. Physiol. Lung Cell. Mol. Physiol. 298, L15-L22 (2010).

16. Peh, W.Y., Reimhult, E., Teh, H.F., Thomsen, J.S. \& Su, X. Understanding ligand binding effects on the conformation of estrogen receptor alpha-DNA complexes: a combinational quartz crystal microbalance with dissipation and surface plasmon resonance study. Biophys J. 92, 4415-4423 (2007).

17. Cao, R., Wang, T.T., DeMaria, G., Sheehan, J.K. \& Kesimer, M. Mapping the protein domain structures of the respiratory mucins: a mucin proteome coverage study. J Proteome Res. 11, 4013-4023 (2012).

18. Raynal, B.D., Hardingham, T.E., Sheehan, J.K. \& Thornton, D.J. Calciumdependent protein interactions in MUC5B provide reversible cross-links in salivary mucus. J. Biol. Chem. 278, 28703-28710 (2003).

19. Rubinstein MaD, A.V. Associations leading to formation of reversible networks and gels. Curr. Opin. Colloid Interface Sci. 4, 83-87 (1999).

20. Chassenieux, C., Nicolai, T. \& Benyahia, L. Rheology of associative polymer solutions. Curr. Opin. Colloid Interface Sci. 16, 18-26 (2011).

21. Verdugo, P. Supramolecular dynamics of mucus. Cold Spring Harb. Perspect. Med. 2, 1-14 (2012).

22. Ridley, C. etal. Assembly of the respiratory mucin MUC5B: a new model for a gel-forming mucin. J. Biol. Chem. 289, 16409-16420 (2014).

23. Meyer, E.E., Rosenberg, K.J. \& Israelachvili, J. Recent progress in understanding hydrophobic interactions. Proc. Natl. Acad. Sci. USA 103, 15739-15746 (2006).

24. Bromberg, L.E. \& Barr, D.P. Self-association of mucin. Biomacromolecules 1, 325-334 (2000).

25. Kesimer, M. \& Sheehan, J.K. Analyzing the functions of large glycoconjugates through the dissipative properties of their absorbed layers using the gel-forming mucin MUC5B as an example. Glycobiology 18, 463-472 (2008).

26. Hayes, J.D. \& Pulford, D.J. The glutathione S-transferase supergene family: regulation of GST and the contribution of the isoenzymes to cancer chemoprotection and drug resistance. Crit. Rev. Biochem. Mol. Biol. 30, 445-600 (1995).

27. Woodward, A.M., Mauris, J. \& Argueso, P. Binding of transmembrane mucins to galectin-3 limits herpesvirus 1 infection of human corneal keratinocytes. J. Virol. 87, 5841-5847 (2013).

28. Argueso, P. Glycobiology of the ocular surface: mucins and lectins. Jpn. J. Ophthalmol. 57, 150-155 (2013).

29. Byrd, J.C. \& Bresalier, R.S. Mucins and mucin binding proteins in colorectal cancer. Cancer Metastasis Rev. 23, 77-99 (2004).

30. Argueso, P., Guzman-Aranguez, A., Mantelli, F., Cao, Z., Ricciuto, J. \& Panjwani, N. Association of cell surface mucins with galectin-3 contributes to the ocular surface epithelial barrier. J. Biol. Chem. 284, 23037-23045 (2009).
31. Seelenmeyer, C., Wegehingel, S., Lechner, J. \& Nickel, W. The cancer antigen CA125 represents a novel counter receptor for galectin-1. J. Cell Sci. 116 (Pt 7), 1305-1318 (2003).

32. Senapati, S., Das, S. \& Batra, S.K. Mucin-interacting proteins: from function to therapeutics. Trends Biochem. Sci. 35, 236-245 (2010).

33. Wiede, A., Jagla, W., Welte, T., Kohnlein, T., Busk, H. \& Hoffmann, W. Localization of TFF3, a new mucus-associated peptide of the human respiratory tract. Am. J. Respir. Crit. Care Med. 159 (4 Pt 1), 1330-1335 (1999).

34. Jagla, W. et al. Secretion of TFF-peptides by human salivary glands. Cell Tissue Res. 298, 161-166 (1999).

35. Kobayashi, K. et al. Distribution and partial characterisation of IgG FC binding protein in various mucin producing cells and body fluids. Gut 51, 169-176 (2002).

36. Johansson, M.E., Thomsson, K.A. \& Hansson, G.C. Proteomic analyses of the two mucus layers of the colon barrier reveal that their main component, the Muc2 mucin, is strongly bound to the Fcgbp protein. J. Proteome Res. 8, 3549-3557 (2009).

37. Kesimer, M. et al. Excess secretion of gel-forming mucins and associated innate defense proteins with defective mucin un-packaging underpin gallbladder mucocele formation in dogs. PLoS One 10, e0138988 (2015).

38. Mclnturff, B.H., Ribeiro, P., Abdullah, C., Kesimer, L. \& Gel-forming, M. mucins in response to infection and inflammation in normal and cystic fibrosis airways. Pediatr. Pulmonol. 50, S225 (2015).

39. Fulcher, M.L., Gabriel, S., Burns, K.A., Yankaskas, J.R. \& Randell, S.H. Well-differentiated human airway epithelial cell cultures. Methods Mol. Med. 107, 183-206 (2005).

40. Pickles, R.J., McCarty, D., Matsui, H., Hart, P.J., Randell, S.H. \& Boucher, R.C. Limited entry of adenovirus vectors into well-differentiated airway epithelium is responsible for inefficient gene transfer. J. Virol. 72, 6014-6023 (1998).

41. Kesimer, M. \& Sheehan, J.K. Mass spectrometric analysis of mucin core proteins. Methods Mol. Biol. 842, 67-79 (2012).

42. Thornton, D.J. et al. Salivary mucin MG1 is comprised almost entirely of different glycosylated forms of the MUC5B gene product. Glycobiology 9, 293-302 (1999).

(c) (1) () $\odot$ This work is licensed under a Creative Commons At 1 Atribution-NonCommercial-NoDerivs 4.0 International License. The images or other third party material in this article are included in the article's Creative Commons license, unless indicated otherwise in the credit line; if the material is not included under the Creative Commons license, users will need to obtain permission from the license holder to reproduce the material. To view a copy of this license, visit http://creativecommons.org/licenses/by-nc-nd/4.0/ 